\title{
Letter to the editor Maternal diseases in pregnancy
}

\author{
Nicolae GICA ${ }^{1,2}$, Gheorghe PELTECU ${ }^{1,2}$, Anca Maria PANAITESCU ${ }^{1,2}$ \\ ${ }^{1}$ Filantropia Clinical Hospital, Bucharest, Romania \\ 2"Carol Davila" University of Medicine and Pharmacy, Bucharest, Romania
}

Pregnancy represents a special period in women's life, with different influences of certain maternal diseases. In the last decades, the obstetrical surveillance and healthcare has changed in Romania, in order to improve neonatal outcomes and to decrease severe obstetric complications but also to reduce the maternal mortality. Severe maternal morbidity may result from the association of multiple risk factor like: maternal age, obesity, chronic medical conditions and cesarean section delivery [1].

There are many diseases with high- risk complication potential during pregnancy, among which the most frequently diagnosed in pregnancy are: hypertensive disorders, diabetes, anemia, thyroid dysfunction and anemia. Hypertensive disorders complications are the second causes of maternal mortality after postpartum hemorrhage. Early diagnosis and treatment can reduce acute fetal complications and maternal cardiovascular, neurological, and renal morbidity.

Moreover, gestational diabetes has a high prevalence in the general population, directly proportional with obesity. Early diagnosis reduces maternal and fetal complications through a correct intervention - dietary measures, insulin administration and fetal monitoring [2].

Despite the high frequency of gestational hypertension [3] and diabetes, there are many other conditions with potential risk in increasing morbidity and mortality in pregnancy. For this reason, we proceed with the proposal of this special issue including the rare and frequent maternal pathologies that can lead to a high-risk pregnancy.

From another point of view, we are writing this letter in order to highlight the importance of early diagnosis and treatment of the associate maternal pathologies with positive impact on pregnancy outcomes. Patients with chronic diseases should undergo preconception examinations and counselling for a correct assessment of drugs administration and correctable risk factors, which might have a greater impact on maternal prognosis, delivery, and the health care system.

Conflict of interest: none declared Financial support: none declared

\section{REFERENCES}

1. Pregnancy Complications. Available at: https://www.cdc.gov/reproductivehealth/ maternalinfanthealth/pregnancycomplications.html.

2. Panaitescu AM, Ciobanu AM, Popa M, et al. Screening for Gestational Diabetes during the COVID-19 Pandemic-Current

Recommendations and Their Consequences. Medicina (Kaunas). 2021;57(4):381.

3. Panaitescu AM, Ciobanu AM, Popescu MR, Huluta I, Botezatu R, Peltecu G, Gica N.
Incidence of hypertensive disorders of pregnancy in Romania. Hypertens Pregnancy. 2020 Nov;39(4):423-428. 